\title{
Formation of Ultrathin Film Between Two Molecularly Smooth Surfaces
}

\author{
M. F. Abd Al-Samieh \\ Mechanical Design \& Production Department, Military Technical College, \\ Cairo, Egypt \\ Email: mohamed.fahmy203@hotmail.com
}

\begin{abstract}
The forces between surfaces determine the properties of many biological systems. This makes them an important field of study. Measurements and numerical work of many researchers showed that in ultrathin lubricating films the net interaction between two surfaces separated by a polar lubricant involves both the electrostatic double layer force and the Van der Waals' force and at small separation solvation pressure is aided. In this work a numerical solution of the Reynolds' equation was developed using NewtonRaphson technique to obtain the film shape and pressure distribution caused by the hydrodynamic viscous action in addition to double layer electrostatic force, Van der Waals' inter-molecular forces and solvation pressure due to inter-surface forces. The numerical results showed that the effect of changing rolling speed and surface potential on the formation of ultrathin lubricating film thickness for polar lubricant confined between two Mica surfaces. The numerical results showed that the film thickness increase by the increasing the rolling speed and surface potential. The increased value of the film thickness is due to the effect of double layer electrostatic force. At small separation the effect of solvation pressure dominates.
\end{abstract}

Keywords: Ultra-thin films, elastohydrodynamics, solvation, Van der Waals' force, electrostatic force

\section{NOMENCLATURE}

$\begin{array}{ll}a & \text { lubricant molecular diameter }=0.5 \mathrm{~nm} \\ A & \text { Hamaker constant }=5.51 \times 10^{-20} \mathrm{Joules} \\ b & \text { radius of Hertzian contact region } \\ C & \text { constant defined in equation }(11)=172 \mathrm{MPa} \\ D & \text { deformation influence coefficient matrix } \\ E_{A, B} & \text { Young's modulus of elasticity } \\ E^{\prime} & \text { reduced modulus of elasticity } \\ G^{*} & \text { materials' parameter, } G^{*}=E^{\prime} \alpha \\ h & \text { lubricant film thickness } \\ H & \text { dimensionless film thickness, } H=h R / b^{2} \\ H_{0} & \text { dimensionless central oil film thickness } \\ l & \text { dimensionless side leakage boundary distance } \\ m & \text { dimensionless inlet distance } \\ n_{x}, n_{y} & \text { number of computational grid nodes } \\ P & \text { total contact pressure } \\ p_{h} & \text { hydrodynamic pressure } \\ p_{s} & \text { solvation pressure due to surfaces' interaction force }\end{array}$




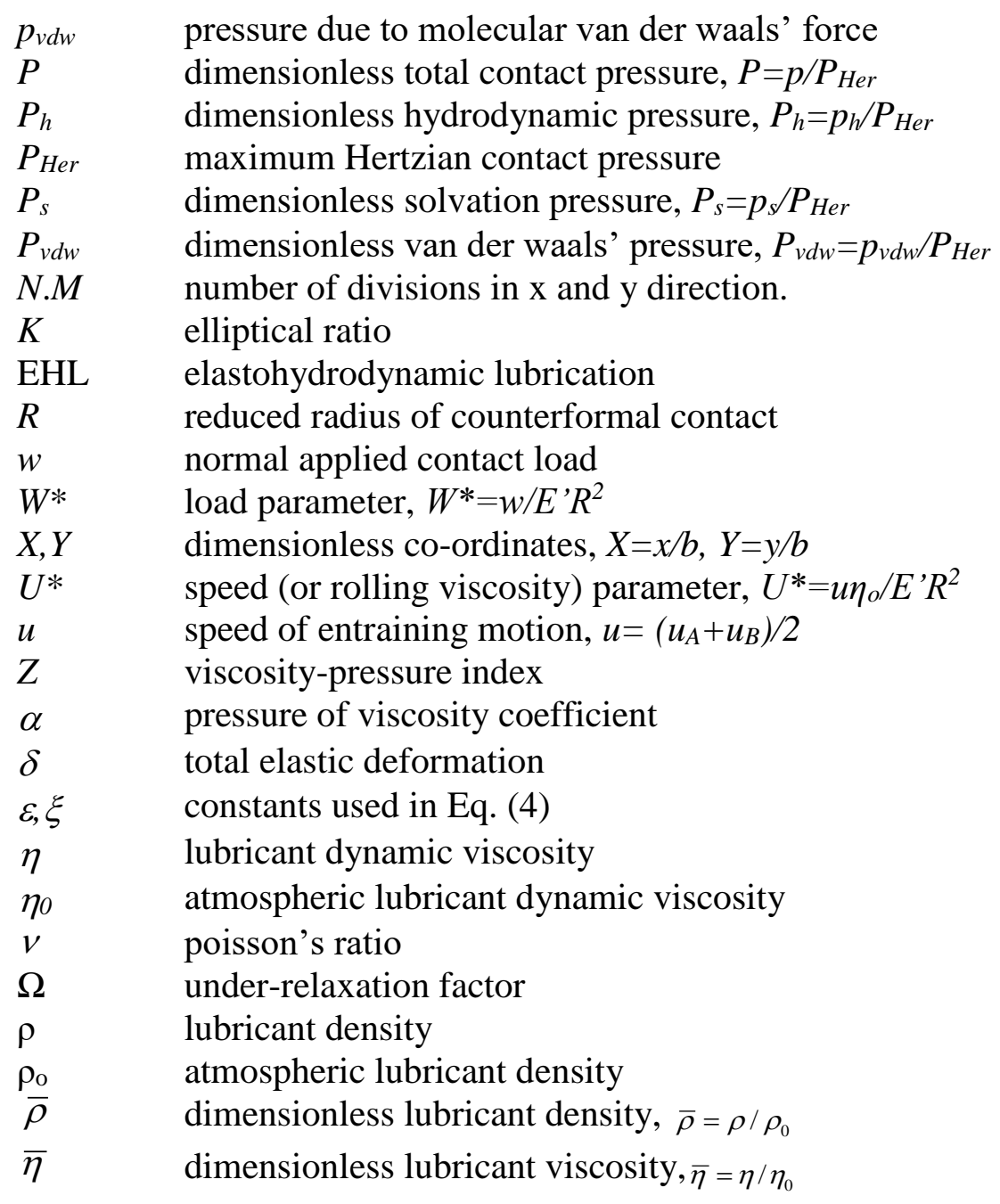

Superscripts:

$i, j \quad$ discrete nodal points in both the $\mathrm{X}$ and $\mathrm{Y}$ directions

$n \quad$ iteration index

Subscripts:

$A, B \quad$ denote the contiguous bodies in contact

$k, l \quad$ covariant influence coefficient indices

\section{INTRODUCTION}

Oil film with a thickness in the nanoscale has been well studied from the beginning of the 1990s. The multi-subject nature of tribology research was enhanced due to rapid development of economy and relative technologies. The foundation of micro/nanotribology is not only a result of the integration of multi-scientific subjects, but also originates from the understanding that a tribology process can proceed across several scales. A reduction in the research scale from macro to micro meter is also determined by the nature of the tribology process itself. In a friction process, for example, the macro tribology property of sliding surfaces depends closely on micro structure or micro interactions on the interface. Therefore, Micro/nanotribology provides a new insight and an innovative research mode. It reveals mechanisms of the friction, wear, and lubrication 
on atomic and molecular scale, or both, and establishes a relationship between the microstructure and macroscopic performance. This is very important for the further development of tribology [1-4].

Israelachvili and Tabor [5] developed the surface force apparatus to measure the Van der Waals' force and later becoming a more advanced one [6] to be well used in the tribological test of thin liquid layer in molecular order. Alsten et al [7], Granick [8] and Luengo et al [9] used surface force apparatus and observed that the adsorptive force between two solid surfaces was strongly related to the distance between the two solid surfaces and the temperature of the lubricant. A number of researcher such as Israelachvili [10], Israelachvili and McGuiggan [11], Christensen and Horn [12] and Chan and Horn [13] have measured the force between two crossed cylinders immersed in a fluid and found that if the lubricant is polar, the force at large separations is well described by the DLVO theory (i.e. the action of Van der Waals' and repulsive double-layer forces together) and below $3 \mathrm{~nm}$ the force is oscillatory.

Johnston et al [14] found that elastohydrodynamic phenomenon did not exist with films less than $15 \mathrm{~nm}$ thick. Tichy [15-17] proposed the models of thin lubricant film according to the improved elastohydrodynamic theory and his results showed that the effective viscosity increases by an order of magnitude near the surfaces due to molecular forces. Hartle et al. [18] have showed that the film thickness of octamethylcyclotetrasiloxane (OMCTS) exhibits a deviation from linearity on a log film thickness versus log rolling speed in the thin film region, which is thicker than that predicted from EHD theory in the low speed region and discretization of both central and minimum film thicknesses can be observed at this low speed (below about $10 \mathrm{~nm}$ ) and the interval of the discretization is approximately $1 \mathrm{~nm}$.

The effect of electric voltage on the film thickness formation in thin film lubrication has been studied by Shen et al [19] and Luo et al [20]. They used hexadecane with the addition of cholesteryl liquid crystals in chemically pure as the lubricant to check the variation of its film thickness by applying an external DC voltage on the lubricant film and found that as the voltage rises and the electrical field becomes stronger, the liquid crystals molecules rearrange in the direction of maximum viscosity, causing an enhancement in film thickness. When the voltage increases further to $500 \mathrm{mV}$, the film thickness reaches a maximum value of about $30 \mathrm{~nm}$. Then the film thickness hardly changes with increasing voltage.

Al-Samieh [21] has studied the behavior of polar lubricants in concentrated contacts in ultra-thin conjunctions. He showed that at separations beyond about five molecular diameters of the intervening liquid, the formation of a lubricant film thickness is governed by combined effects of viscous action and surface force of an attractive Van der Waals' force and a repulsive double layer force. At smaller separations below about five molecular diameters of the intervening liquid, the effect of solvation force is dominant in determining the oil film thickness and the discretization of the film thickness occurs at this low speed region.

This paper attempts to contribute to the on-going process of understanding of ultra-thin film lubrication formation by investigating the relationship between the speed of entraining motion and the film thickness and to investigate the relationship between the surface potential and the film thickness under the condition where surface force action of solvation, Van der Waals as well as electrostatic force are included, where the intervening liquid between the two planner surfaces is polar. 


\section{BACKGROUND THEORY}

In the case of ultra-thin film thickness the total pressure caused by the Van der Waals; $P_{v d w}$, solvation; $P_{s}$, and double layer electrostatic force; $P_{e}$, in addition to the conventional viscous pressure; $P_{h}$ must be taken into consideration when calculating the film thickness. Therefore, the total pressure $P$ is given as:

$$
P=P_{s}+P_{v d w}+P_{e}+P_{h}
$$

\section{Elastohydrodynamic Pressure}

The Reynolds' equation in dimensionless form under steady-state entraining motion can be written as in Eq. (2):

$$
\frac{\partial}{\partial X}\left(\frac{\bar{\rho} H^{3}}{\bar{\eta}} \frac{\partial P_{h}}{\partial X}\right)+\frac{1}{K^{2}} \frac{\partial}{\partial Y}\left(\frac{\bar{\rho} H^{3}}{\bar{\eta}} \frac{\partial P_{h}}{\partial Y}\right)=\lambda \frac{\partial}{\partial X}(\bar{\rho} H)
$$

where;

$$
\begin{array}{ll}
X=x / b, & Y=y / a \\
\bar{\eta}=\eta / \eta_{0}, \bar{\rho}=\rho / \rho_{0}, & \lambda=\frac{12 u \eta_{o} R_{x}^{2}}{b^{3} P_{\mathrm{Her}}}, \\
P h=p h / p H e r, & H=h R_{x} / b 2,
\end{array}
$$

The density of the lubricant variation with pressure is given by Dowson and Higginson [22] as Eq. (3).

$$
\bar{\rho}=1+\frac{\varepsilon P_{h} P_{H e r}}{1+\xi P_{h} P_{H e r}}
$$

where $\varepsilon$ and $\zeta$ are constants, dependent upon the type of lubricant used.

The Roelands [23] gives the equation expressing the influence of pressure upon viscosity. This equation can be written in non-dimensional terms in the form of Eq. (4).

$$
\bar{\eta}=\exp \left[\ln \eta_{\circ}+9.67\right]\left[\left(1+5.1 * 10^{-9} P_{h} P_{H e r}\right)^{Z}-1\right]
$$

where:

$$
\mathrm{Z}=\frac{\alpha}{5.1 * 10^{-9}\left[\ln \eta_{\circ}+9.67\right]}
$$

The dimensionless form of film shape equation is assumed to be of the same as that reported by Hamrock and Dowson [24], given by Eq. (5). 


$$
H(X, Y)=H_{\circ}+\frac{(X-m)^{2}}{2}+\frac{K^{2} R_{x}}{R_{y}} \frac{(Y-l)^{2}}{2}+\frac{R_{x} \delta(X, Y)}{b^{2}}
$$

where, the dimensional elastic deformation at any point $\mathrm{x}, \mathrm{y}$ is defined by Hamrock and Dowson [24] is in Eq. (6).

$$
\delta_{I, J}(x, y)=\frac{2}{\Pi} \sum_{j=1}^{n y} \sum_{i=1}^{n x} P_{i, j} D_{i^{*}, j^{*}}
$$

Where, $\quad i^{*}=|I-i|+1, j^{*}=|J-j|+1$

The Newton-Raphson method is applied to the Reynolds'equation in the following numerical form of Eq. (7).

$$
\sum_{l=2}^{N} \sum_{k=2}^{M} \frac{\partial F_{i, j}}{\partial P_{h k, l}} \Delta P_{h k, l}=-F_{i, j}
$$

where, $i, j$ are the discrete nodal points in both the $\mathrm{X}$ and $\mathrm{Y}$ directions, $M, N$ are the number of divisions in $\mathrm{X}$ and $\mathrm{Y}$ directions respectively, $k=1,2, \ldots . M, l=1,2, \ldots, \mathrm{N}$ and the Jacobian matrix given in terms of the residual derivatives as in Eq. (8).

$$
J_{k, l}^{i, j}=\frac{\partial F_{i, j}}{\partial P_{k, l}}
$$

The system state equation can be written using the Gauss-Seidel iteration method as Eq. (9).

$$
\Delta P_{k, l}^{n}=\left(-F_{i, j}-J_{k-1, l}^{i, j} \Delta P_{k-1, l}^{n}-J_{k+1, l}^{i, j} \Delta P_{k+1, l}^{n-1}-J_{k, l-1}^{i, j} \Delta P_{k, l-1}^{n}-J_{k, l+1}^{i, j} \Delta P_{k, l+1}^{n-1}\right) / J_{k, l}^{i, j}
$$

where $\mathrm{n}$ is the iteration counter in the above recursive equation.

For the reason of good numerical stability an under-relaxation factor $(\Omega)$ typically chosen as 0.01 is employed to update the pressure according to Eq. (10).

$$
P_{i, j}^{n}=P_{i . j}^{n-1}+\Omega \Delta P_{i, j}^{n}
$$

The pressure convergence criterion is given as:

$$
\left[\frac{\sum_{i} \sum_{j}\left(\bar{P}_{i, j}^{n}-\bar{P}_{i, j}^{n-1}\right)^{2}}{N}\right]^{0.5} \leq 10^{-4}
$$


while the load balance convergence criterion is given by:

$$
\left|\iint P(X, Y) d X d Y-\frac{2}{3} \pi\right| \leq 10^{-4}
$$

\section{Molecular and Surface Actions in Narrow Conjunctions}

In polar lubricant, the interactions between solid surfaces consist mainly of three important forces: Van der Waals, solvation and electrostatic double layer forces.

\section{Solvation Pressure}

Whenever liquid molecules are introduced into a highly restricted gap and are forced to form a series of layers a solvation pressure occurs. This force takes an oscillatory and exponentially decaying function of separation according to molecular ordering in the gap. Israelachvili [6], Israelachvili et al [25], Horn and Israelachvili [26], Van Balderas Altamiranoa and Gama Goicochea [27] and John Everett and David Faux [28] have studied the solvation force in the narrow contact of contiguous bodies. The solvation pressure is given by Israelachvili [6] is in Eq. (11).

$$
P_{s}=-C e^{\frac{-h}{a}} \cos (2 \pi h / a)
$$

\section{Van der Waals' Pressure}

Many researchers [6, 27 and 29] studied the Van der Waals' force between two surfaces when they are separated by a very thin liquid film. They found that, the pressure due to the Van der Waals' forces can be written as Eq. (12):

$$
P_{v d w}=\frac{-A}{6 \pi h^{3}}
$$

\section{Electrostatic Forces between Surfaces}

As shown by many researchers [30-32] the electrostatic force between two charged surfaces decays roughly exponentially with the distance. The repulsive electrostatic pressure between two surfaces can be written as Eq. (13).

$$
p_{\text {elest }}=64 K T \rho_{\infty} \chi^{2} e^{-\kappa h}
$$

where, $\chi=\tanh \left(z e \psi_{o} / 4 K T\right), z$ is the valency, $e$ is the electronic charge, $\psi_{o}$ is the electrostatic surface potential, $K$ is the Boltzmann constant, $T$ is the temperature in degrees Kelvin, $\rho_{\infty}$ is the electrolyte concentration in the bulk and $\kappa$ is the Debye length. At less than $25 \mathrm{mV}$, the above Eq. (13) can be simplified as shown by Israelachvili [6] to Eq. (14). 


$$
p_{\text {elest }}=2 \varepsilon \varepsilon_{o} \kappa^{2} \omega_{0}^{2} e^{-\kappa h}
$$

where, $\varepsilon$ is the dielectric constant of the liquid (for propylene carbonate $=65$ ), ${ }^{\varepsilon_{o}}$ is the dielectric permittivity of the free space ( $8.854 \times 10^{-12}$ (farads / meter), $k$ is the Debye length and $\omega_{0}$ is the surface potential.

\section{RESULTS AND DISCUSSION}

In this study, the solutions of the film thickness and the pressure in the lubricated contacts are solved numerically using the properties of lubricants and contacting materials given in Table 1. The lubricant used in the simulation study is propylene carbonate. This lubricant has a rigid molecular diameter of $0.5 \mathrm{~nm}$. Newton-Raphson method is used for the solution of the Reynolds' equation to obtain the film shape and pressure distribution. The numerical computing procedure of simultaneous elastic deformation and lubricant pressures is the same for all the cases discussed in the paper. A nodal density in the range of 10000-60000 is used for the evaluation of pressure and elastic deformation. The load and speed of entraining motion are chosen in such that they promote the formation of ultrathin lubricating film thickness to see the effect of changing the rolling speed and surface potential. The validation of the numerical method employed in the paper is explained in more detail by Al-Samieh [21] and the results showed that very good agreement for the numerical results and that published by Kato and Matsuoka [33].

Table 1. Physical and geometrical properties of contacting materials and PC lubricant

\begin{tabular}{clc}
\hline Designation & Parameters & Values \\
\hline$\eta o$ & Viscosity & $2.8 \mathrm{mPa} . \mathrm{s}$ \\
$a$ & Molecular diameter & $0.5 \mathrm{~nm}$ \\
$A$ & Hamaker constant & $5.51 \times 10-20 \mathrm{Joules}$ \\
$E_{A}$ & Young's modulus & $34.5 \mathrm{GPa}$ \\
$E_{B}$ & Young's modulus & $34.5 \mathrm{GPa}$ \\
$v_{A}$ & Poisson's ratio & 0.205 \\
$v_{B}$ & Poisson's ratio & 0.205 \\
$\alpha$ & Pressure of viscosity coefficient & $10 \mathrm{GPa}^{-1}$ \\
$R_{A x}$ & Radius of curvature of solid A in x-direction & $0.01111 \mathrm{~m}$ \\
$R_{A y}$ & Radius of curvature of solid A in y-direction & $0.01111 \mathrm{~m}$ \\
$R_{B x}$ & Radius of curvature of solid B in x-direction & $\infty \mathrm{m}$ \\
$\varepsilon$ & Constant used in equation (3) & $5.83 \times 10^{-10} \mathrm{~Pa}$ \\
$\xi$ & Constant used in equation (3) & $1.68 \times 10^{-9} \mathrm{~Pa}$ \\
\hline
\end{tabular}

\section{Effect of Changing Rolling Speed on The Formation of Ultrathin Lubricant Film}

In order to investigate the relationship between the rolling speed and the formation of ultrathin lubricating film thickness a number of simulations study has been carried out for different speed of entraining motion varying from (50-1000) $\mu \mathrm{m} / \mathrm{s}$. Figures 1 and 2 show the variation of film thickness with the rolling speed for $\mathrm{W}^{*}=1.388 \times 10^{-11}(0.05 \mathrm{mN})$ and $\mathrm{W}^{*}=2.381 \times 10^{-11}(0.1 \mathrm{mN})$ for $\mathrm{G}^{*}=360$ and surface potential of $25 \mathrm{mV}$ respectively. It is 
assumed that, the hydrodynamic theory can maintain down to a very low speed and the film thickness can be estimated, using either Brewe el al [34] or Hamrock and Dowson's [35] extrapolated oil film thickness equations, both for elliptical point contact geometries under iso-viscous rigid or iso-viscous elastic regime of lubrication respectively. As shown in Figure 1 and 2 respectively, when the rolling speed of contacting surfaces decreases to about $\mathrm{U}^{*}=3.887 \times 10^{-16}(50 \mu \mathrm{m} / \mathrm{s})$ and to about $\mathrm{U}^{*}=7.775 \times 10^{-16}(100 \mu \mathrm{m} / \mathrm{s})$, the film thickness exhibits a deviation from linearity and the relationship between film thickness and speed of entraining motion becomes much weaker than that in EHD, which is thicker than that predicted from EHD theory in the low speed region, i.e., the thinner the film is, the less the correlation between velocity and bulk viscosity will be. In this region, the film thickness seems to stabilize around a limiting value even if the surface speed decreases and then jumps down suddenly to the next stable thickness when the surface speed amounts to some enough value. This means that, the dominant film forming mechanism in this regime is due to the action of structural forces solvation. The physical explanation for the increased values of the oil film thickness in this region is due to the action of strong interaction between metal surfaces and a polar lubricant as shown by a number of researchers such as Al-samieh [21], Kato and Matsuoka [33], Al-samieh and Rahnejat [36], Chu et al [37] and Prakash Chandra Mishra [38].

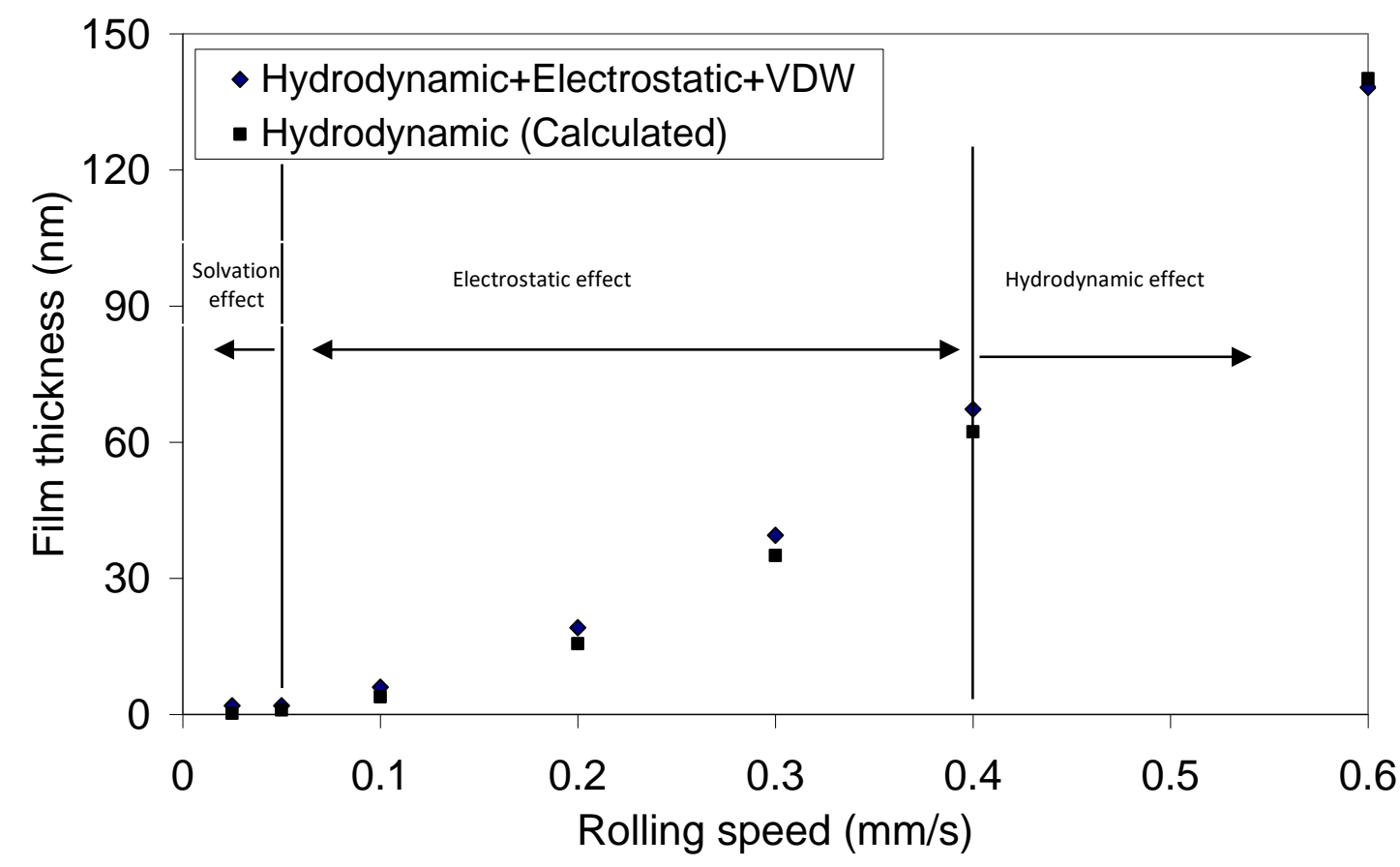

Figure 1. Variation of film thickness with rolling speed for $\mathrm{W}^{*}=1.388 \mathrm{X} 10^{-11}$

As the speed of entraining motion increased above that of $U^{*}=3.887 \times 10^{-16}(50$ $\mu \mathrm{m} / \mathrm{s})$, and above that of $U^{*}=7.775 \times 10^{-16}(100 \mu \mathrm{m} / \mathrm{s})$ as shown in figures 1 and 2 respectively, the relationship between the film thickness and speed of entraining motion is in liner style follow the same pattern of hydrodynamic lubrication theory. The only difference is the increased the value of the numerically obtained film thickness from the current numerical analysis than that the value calculated using either Brewe el al [34] or Hamrock and Dowson's [35] extrapolated oil film thickness equations, both for elliptical point contact geometries under iso-viscous rigid or iso-viscous elastic regime of 
lubrication respectively in the region of film thickness below than about $60 \mathrm{~nm}$. This increase in film thickness value is attributed to the fact that, the film thickness formation in this region is caused due to the combined effect of hydrodynamic action and the effect of Van der Waals' and the electrostatic double layer forces action. Therefore, the effect of Van der Waals' and the electrostatic double layer forces causes the film thickness to be increased by about 20 to $25 \%$ of the actual film. This means that, the increased in lubricant film thickness is caused due to the fact that, a repulsive action of the electrostatic force is greater than an attractive value of Van der Waals and this net repulsive value is aided to the hydrodynamic action and this leads to the increased in the film thickness.

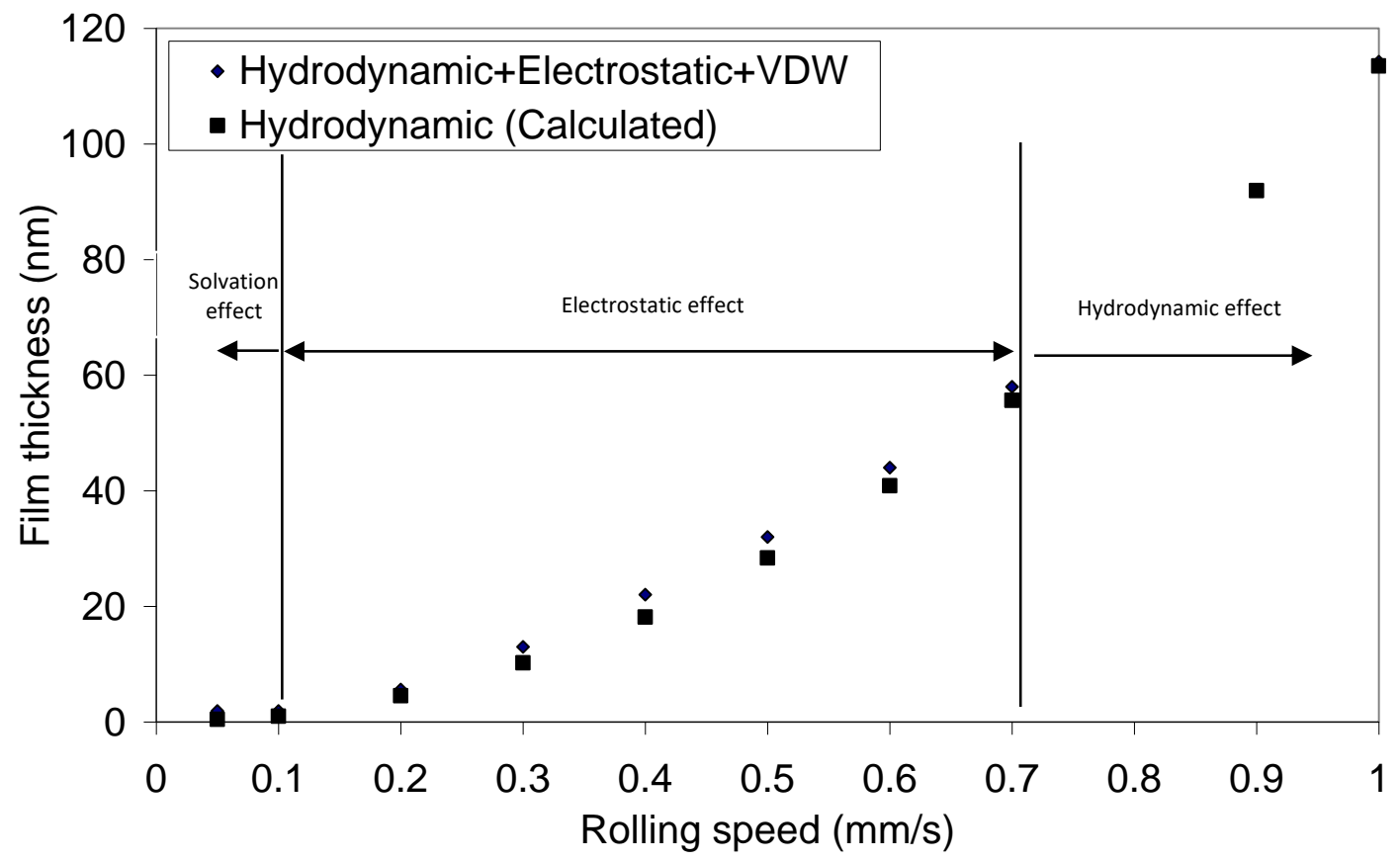

Figure 2. Variation of film thickness with rolling speed for $\mathrm{W}^{*}=2.381 \times 10^{-11}$

Figure 3 (a) and (b) show the dimensionless pressure profile $\mathrm{P}$ and the associated dimensionless film thickness $\mathrm{H}$ in the direction of entraining motion through the central film at constant applied load of $W^{*}=2.381 \times 10^{-11}(0.1 \mathrm{mN})$ and $\mathrm{G}^{*}=360$ for $\mathrm{U}^{*}=3.599 \mathrm{x}$ $10^{-16}(50 \mu \mathrm{m} / \mathrm{s})$ and $U^{*}=3.599 \times 10^{-15}(500 \mu \mathrm{m} / \mathrm{s})$ respectively. Both figures show the wellknown features for lightly loaded contacts. The pressure profile shown in Figure 3 (a) deviates from the conventional hydrodynamic pressure in that the oscillatory solvation pressure is aided to the conventional hydrodynamic viscous pressure, where the solvation pressure has an oscillatory repulsive-attractive nature. According to the Greenwood chart [39], the condition of this example falls into Rigid-Isoviscos region where the elastic deformation is negligible, therefore, the flattened shape of the film thickness shown in Figure 3 (a) is due to the effect of solvation pressure and the dominant film forming mechanism in this case is due to action of structural forces. In fact, the film thickness in this case equal to $1.89 \mathrm{~nm}$ compared to the value of $0.434 \mathrm{~nm}$ calculated from extrapolated oil film thickness equations of Brewe el al [34] for elliptical point contact geometries under iso-viscous rigid regime of lubrication. Therefore, the solvation force causes the film thickness to be increased by about $75 \%$ in this example. Therefore, in these narrow conjunctions the use of extrapolated formulae is inappropriate. 


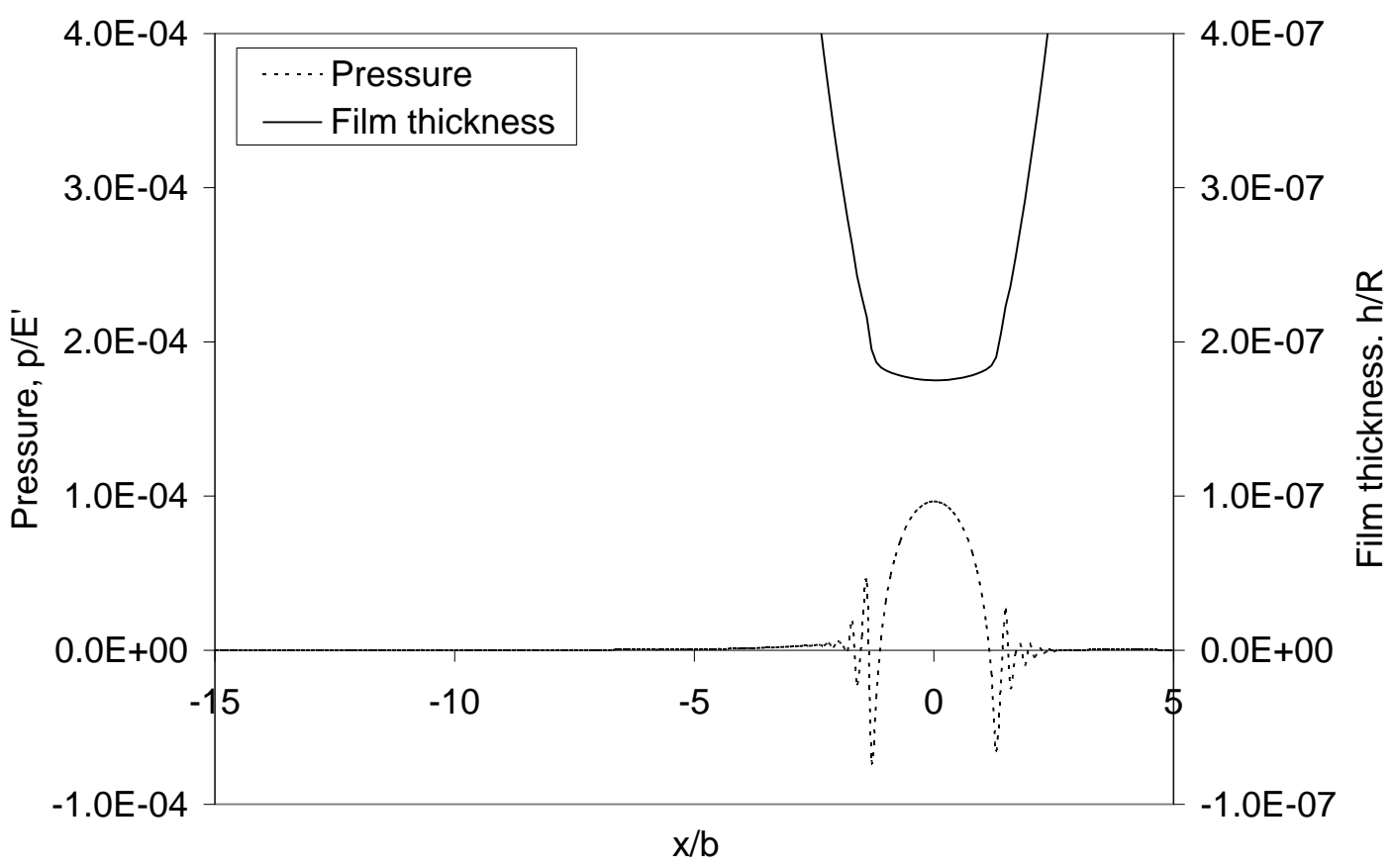

(a)

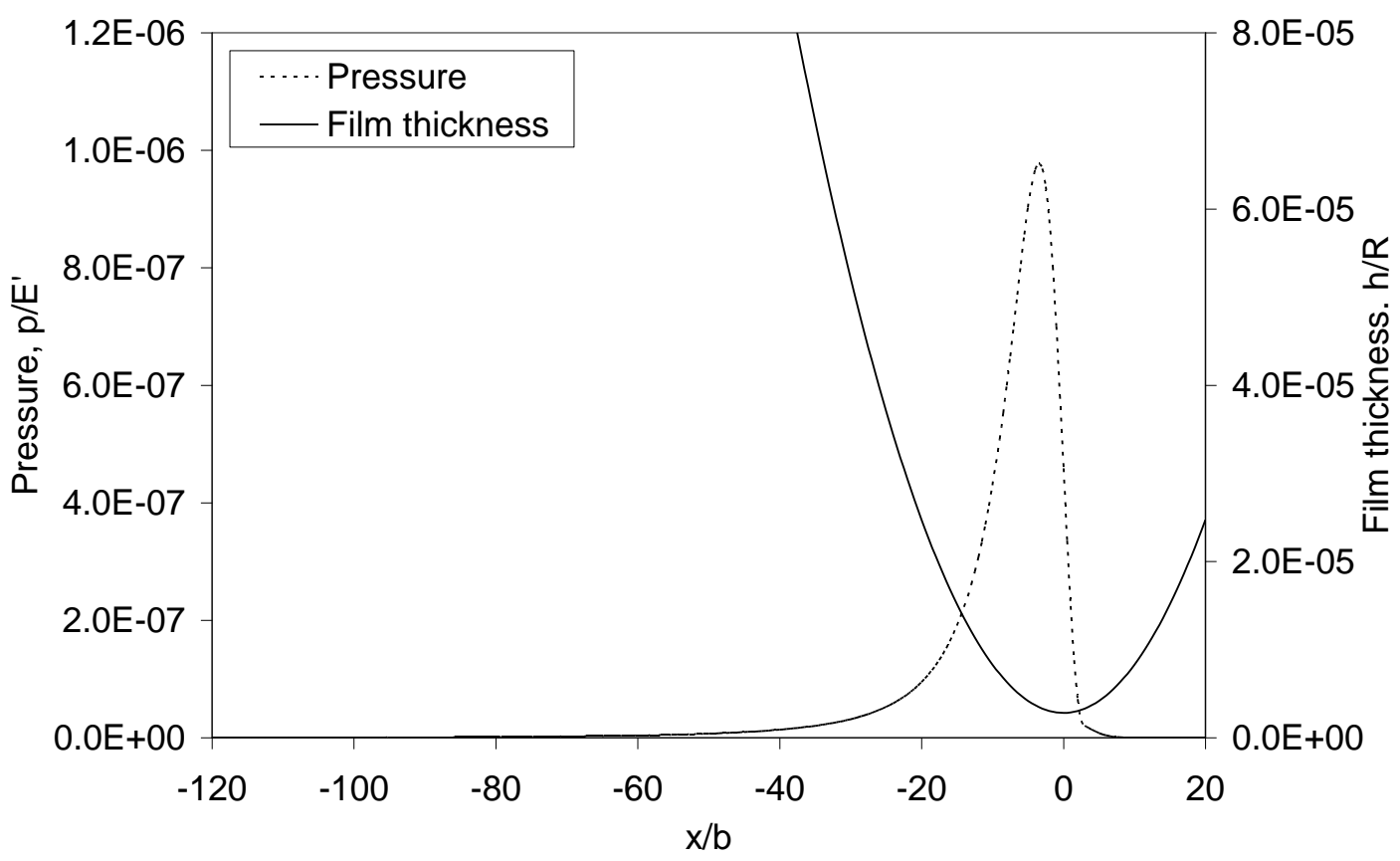

(b)

Figure 3. Total pressure profile and film shape in the central line of contact as the result of combined viscous action and surface force of solvation and Van der Waals forces and electrostatic double layer forces for $\mathrm{W}^{*}=2.381 \times 10^{-11}$, (a) $\mathrm{U}^{*}=3.599 \times 10^{-16}$ and (b) $\mathrm{U}^{*}=3.599 \times 10^{-15}$.

The pressure profile and film thickness shape in the direction of entraining motion through the central film shown in Figure 3 (b) approximate the conventional hydrodynamic pressure and film thickness shape. In this region the effect of hydrodynamic pressure is quite significant and according to the Greenwood chart [39], 
the condition of this example falls into Rigid-Isoviscos region. The film thickness obtained under these conditions of the effect of Van der Waals' and the electrostatic double layer forces (i.e. the action of DLVO theory) is equal to $32.98 \mathrm{~nm}$ compared to the calculated value of film thickness using Brewe el al [34] extrapolated oil film thickness equations of 28.37 (i.e. the film thickness increased by about $16 \%$.). This increased in film thickness is due to the effect of electrostatic double layer force.

\section{Effect of Changing Surface Potential on The Formation of Ultrathin Lubricant Film}

In order to investigate the relationship between the surface potential and the formation of ultrathin film thickness a number of a computational study has been carried out for values of surface potential varied from $(5-25) \mathrm{mV}$. The variation of film thickness against applied load is shown in Figure 5 for different values of surface potential and for speed of entraining motion of $U^{*}=7.199 \times 10^{-16}(100 \mu \mathrm{m} / \mathrm{s})$. It can be observed from this figure that the energy barrier increased as the surface potential was increased. This appeared by that, increasing the values of the surface potential of the surfaces, the values of the minimum film thickness increased for the same values of the applied load. Under the condition of surface potential of $25 \mathrm{mV}$, the film thickness obtained is higher than those obtained for values of surface potential of $5,10.15 \mathrm{mV}$ for the same values of the applied load. This case can be explained as the increased the values of the electrostatic double layer force with the increasing the surface potential. At high values of surface potential, the double-layer repulsion is strong enough to keep the surfaces apart. With decreasing the surface potential, the electrostatic repulsion is more and more screened. At a certain value of surface potential, the Van der Waals' attraction overcomes the repulsive electrostatic barrier and the two surfaces adhered together. This case appears for loads greater than $0.05 \mathrm{mN}$ for surface potential below than $25 \mathrm{mN}$. In fact, the film thickness decreases with load in all the cases as in the same pattern as shown previously using either Brewe el al [34] or Hamrock and Dowson's [35] extrapolated oil film thickness equations, both for point contact geometries under iso-viscous elastic or iso-viscous rigid regime of lubrication respectively.

Figure 6 shows the variation of electrostatic and Van der Waals' pressures with distance for a surface potential of $25 \mathrm{mV}$ for the load of $0.05 \mathrm{mN}$ and speed of entraining motion of $100 \mu \mathrm{m} / \mathrm{s}$. It is clear that, the extent of the electrostatic double layer and Van der Waals pressures decrease with increase in distance. At small separation, the Van der Waals' force dominates causes the two surfaces to be stick together. Fortunately, under the previously mentioned condition, this is not happened, because the surfaces separation is caused due to the effect of hydrodynamic action. On the other hand, the electrostatic double layer repulsion is increased at larger separations cause the separation between the two surfaces to be increased in comparison to the effect of hydrodynamic action alone. By increasing the applied load, the film thickness decreases caused that the value of the Van der Waals' force overcome the value of the hydrodynamic action and that of electrostatic double layer forces causes the film thickness to be collapsed. 


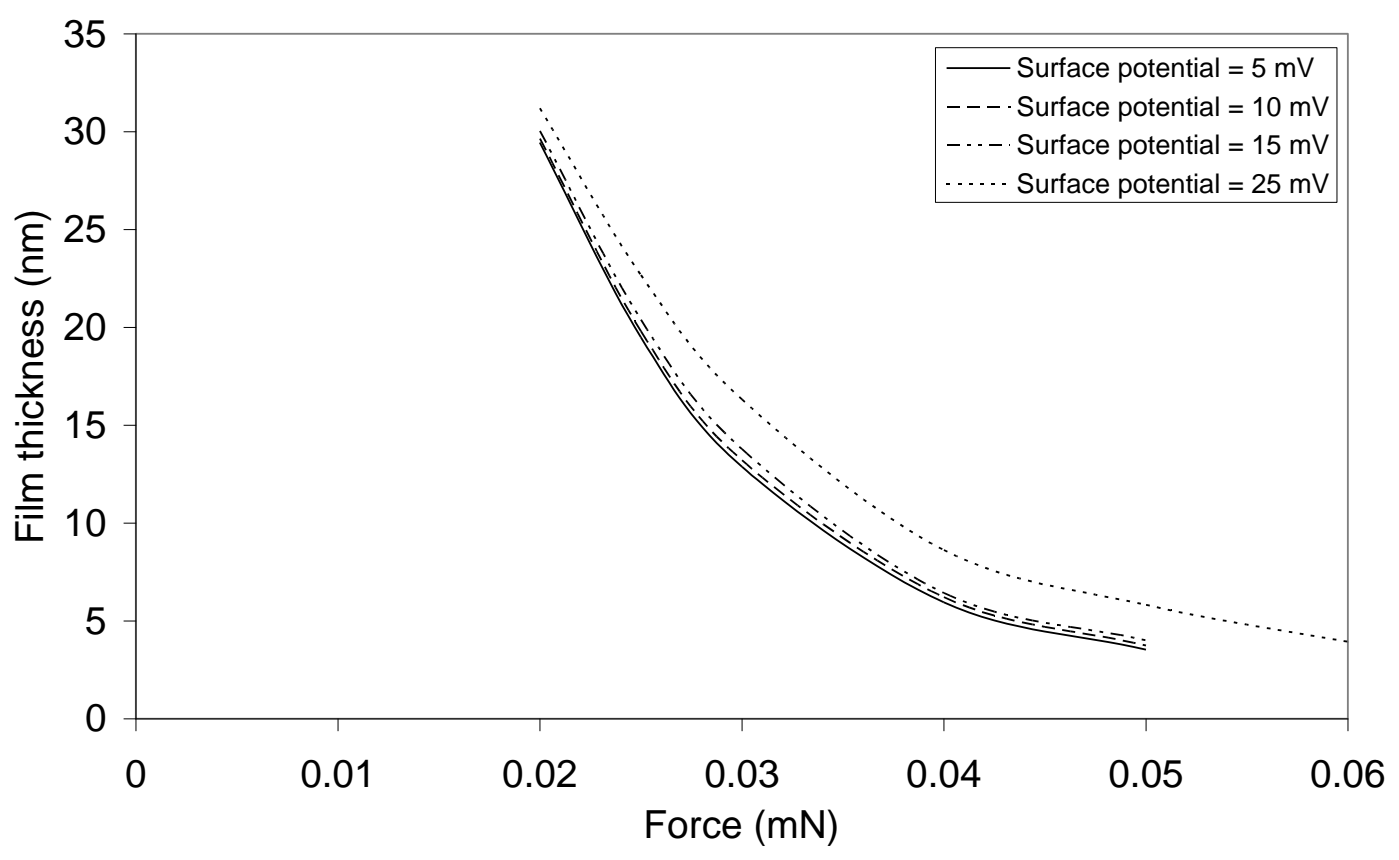

Figure 5. Variation of film thickness with load for different values of surface potential

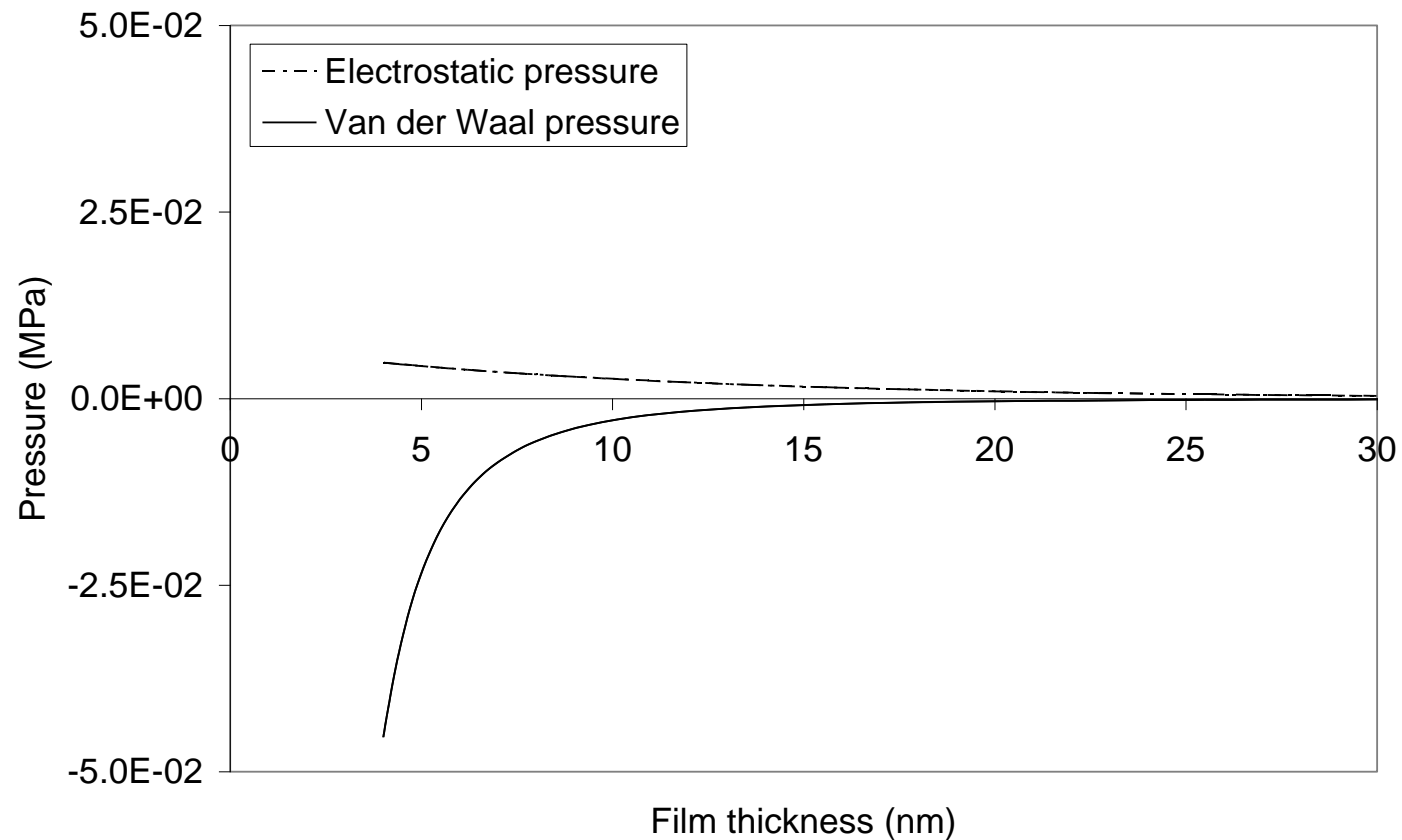

Figure 6. Variation of electrostatic pressure and Van der Waal pressure against film thickness for surface potential of $25 \mathrm{mV}$

Figure 7 shows the effect of variation of surface potential on the electrostatic interaction pressure for the load of $0.025 \mathrm{mN}$ and speed of entraining motion of $100 \mu \mathrm{m} / \mathrm{s}$. It can be observed from this figure that the extent of electrostatic pressure barrier increased as the potential was increased. At very low values of surface potential (say $5 \mathrm{mV}$ ) the electrostatic pressure value drops, and it has a little effect on the film thickness formation. For speed of entraining motion of $100 \mu \mathrm{m} / \mathrm{s}$ and applied load of 
$0.025 \mathrm{mN}$, the aforementioned conditions fall into Rigid-Isoviscos region in the Greenwood chart [39] and the film thickness calculated using Brewe et al [34] formula for circular point contacts under iso-viscous rigid regime of lubrication is $18.16 \mathrm{~nm}$ while that obtained by adding the electrostatic force with surface potential of $5 \mathrm{mV}$ is $19.49 \mathrm{~nm}$, and that of $10 \mathrm{mV}$ is $19.80 \mathrm{~nm}$ and that of $15 \mathrm{mV}$ is $20.39 \mathrm{~nm}$ and that of $25 \mathrm{mV}$ is 22.67 $\mathrm{nm}$ i.e the film thickness increased due to the action of electrostatic pressure by approximately $7 \%, 9 \%, 12 \%$ and $25 \%$ respectively.

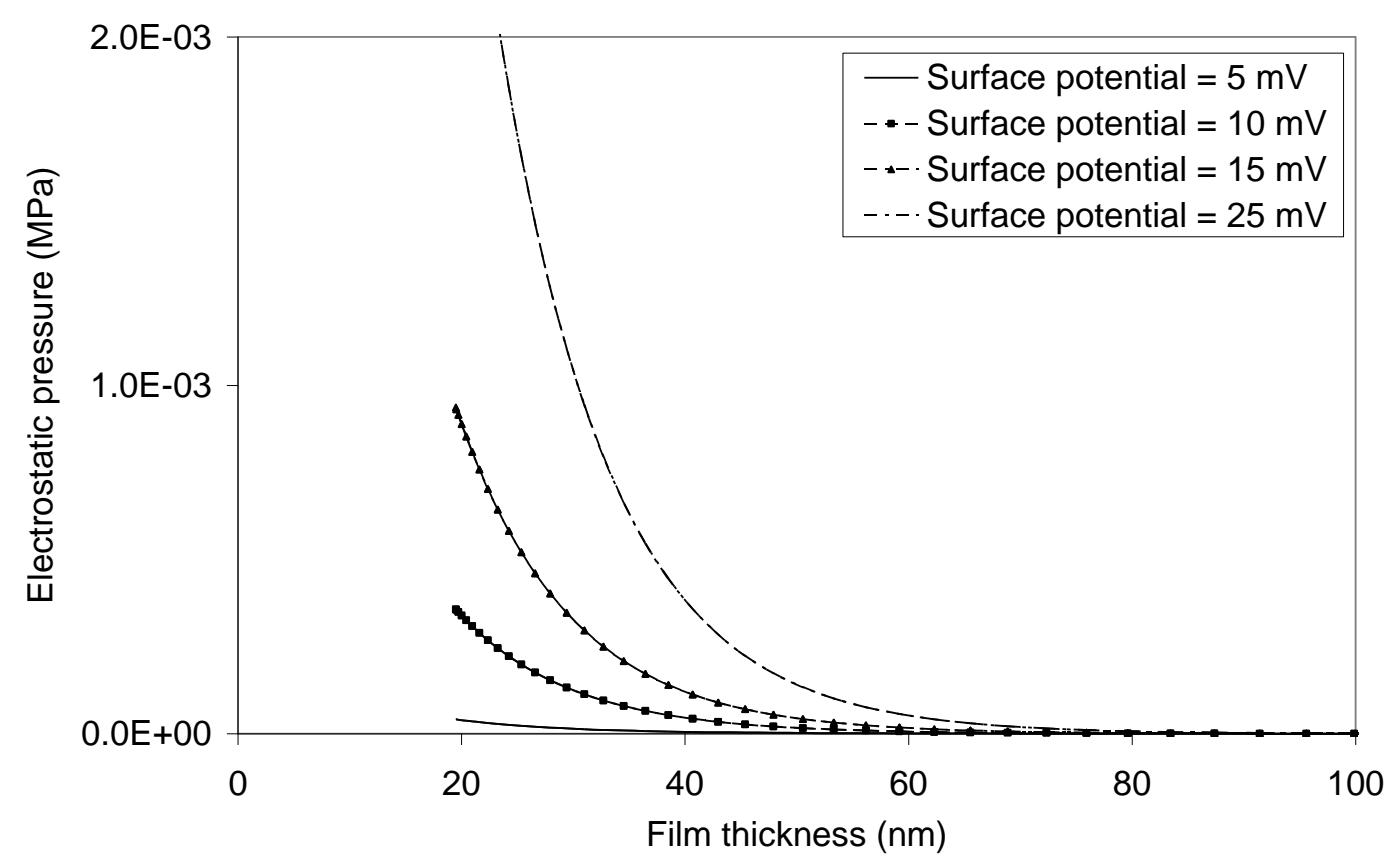

Figure 7. Variation of Electrostatic pressure against film thickness for different values of surface potential for load of $0.025 \mathrm{mN}$ and speed of $100 \mu \mathrm{m} / \mathrm{s}$

\section{CONCLUSION}

The main result of simulations is to show the transition of the ultrathin film between region dominated by the solvation effect and that dominated by electrostatic double layer forces and also to show the effect of changing the surface potential on the formation of ultrathin lubricating film. The film thickness increased with speed follow the same pattern of either Brewe el al [34] or Hamrock and Dowson's [35] extrapolated oil film thickness equations, both for elliptical point contact geometries under iso-viscous rigid or isoviscous elastic regime of lubrication respectively. When the film thickness decreases to about $60 \mathrm{~nm}$ with reducing speed, the film thickness is larger than that calculated from hydrodynamic theory either of Brewe el al [34] or Hamrock and Dowson's [35]. This increased in value of the film thickness is attributed to the effect of electrostatic force and the results are in agreement with that shown by Israelachvili [6], Israelachvili and Mcguiggan [11] and Christensen and Horn [12]. As the speed of entrain motion is further reduced, the film thickness value is greater than that calculated either of the formulas mentioned above. This increased value of the film thickness is attributed to the effect of the solvation pressure. The results also showed that, the extent of repulsive pressure of electrostatic double layer force is increased against distance when the surface potential increased. 


\section{REFERENCES}

[1] Wang X, Guo F, Yang P. A measurement system for thin elastohydrodynamic lubrication films. Frontiers of Mechanical Engineering in China. 2007; 2: 193196.

[2] Dong Z. On some aspects of numerical solutions of thin-film and mixed elastohydrodynamic lubrication. Proceedings of the Institution of Mechanical Engineers Part J: Journal of Engineering Tribology. 2007; 221: 561- 579.

[3] Kalle L, Niko G, Alexander E, Helge L. Real-Time IR Study of Ultra-Thin Film Photopolymerization of Liquid Porphyrin Monomer. Macromol. Rapid Commun. 2010; 31: 1977-1980.

[4] Ohmae N, Martin JM, Mori S. Micro and Nanotribology. TJ1075.O36, ASME Press, New York; 2005.

[5] Israelachvili JN, Tabor D. The Measurement of Van der Waals Dispersion Force in the Range of $1.5 \mathrm{~nm}$ to $130 \mathrm{~nm}$. Proc. R. Soc. London, Ser. A. 1972; 331: 1938.

[6] Israelachvili J. Intermolecular and Surface Force, 2nd ed., Academic Press, San Diego, CA; 1991

[7] Alsten JV, Granick S. Friction Measured with a Surface Forces Apparatus. Tribol. Trans. 1989: 32: 246-250.

[8] Granick S. Motions and Relaxation of Confined Liquid. Science. 1991; 253: 1374-1379.

[9] Luengo G, Schmitt F, Hill R. Thin Film Rheology and Tribology of Confined Polymer Melts: Contrasts with Bulk Properties. Macromolecules. 1997; 30: 24822494.

[10] Israelachvili JN. Solvation forces and liquid structure, as probed by direct force measurements. Acc. Chemical Res. 1987; 20: 415-421.

[11] Israelachvili JN, Mcguiggan PM. Forces between surfaces in liquids. Science. 1988; 241: 795-800.

[12] Christensen HK, Horn RG. Direct measurement of the force between solid surfaces in a polar liquid. J. Chemical physical letters. 1983; 98: 45-48.

[13] Chan DYC, Horn R.G. The drainage of thin liquid films between solid Surfaces. J. Chem. Phys. 1984; 83: 5311-5324.

[14] Johnston GJ, Wayte R, Spikes HA. The Measurement and Study of Very Thin Lubricant Films in Concentrate Contact. STLETribol. Trans. 1991;34: 187-194.

[15] Tichy JA. Modeling of Thin Film Lubrication. STLE Tribol. Trans. 1995; 38: 108-118.

[16] Tichy JAA. Surface Layer Model for Thin Film Lubrication. STLE Tribol. Trans. 1995; 38: 577-582.

[17] Tichy JAA. Porous Media Model of Thin Film Lubrication. Trans.ASME,J. Tribol. 1995; 117: 16-21.

[18] Hartl M, K1upka I, Liška M. Experimental Study of Boundary Layers Formation by Thin Film Colorimetric Interferometry. Sci. China, Ser. A: Math., Phys., Astron. Technol. Sci. 2001; 44: 412-417.

[19] Shen MW, Luo JB, Wen SZ, Yao JB. Nano- Tribological Properties and Mechanisms of the Liquid Crystal as an Additive. Chinese Science Bulletin, 2001; 46: 1227-1232.

[20] Luo JB, Shen MW, Wen SZ. Tribological Properties of Nanoliquid Film under an External Electric Field. J. Appl. Phys. 2004; 96: 6733-6738. 
[21] Al-samieh MF. Film thickness formation in nanoscale due to effects of Elastohydrodynamic, Electrostatic and Surface force of Solvation and Van der Waals. Tribology in industry. 2017; 39: 120-128.

[22] Dowson D, Higginson GR. A numerical solution to the elastohydrodynamic problem. J. Mech. Eng. Sci. 1959; 1: 6-15.

[23] Roelands CJA. Correlation aspects of viscosity-temperature-pressure relationship of lubricating oils. PhD thesis, Delft University of Technology, The Netherlands; 1966.

[24] Hamrock BJ, Dowson D. Isothermal elastohydrodynamic lubrication of point contact. Part I- theoretical formulation. Trans.ASME, J. Tribology. 1976; 98: 223229.

[25] Israelachvili JN, Mcguiggan PM, Homola AM. Dynamic properties of molecularly thin liquid films. Science. 1988; 240: 189-191. 